\title{
BeppoSAX observations of the quasar Markarian 205
}

\author{
P. Favre ${ }^{1,2}$, P.-O. Petrucci ${ }^{3}$, V. Beckmann ${ }^{4,5}$, and T. J.-L. Courvoisier ${ }^{1,2}$ \\ 1 INTEGRAL Science Data Centre, 16 Ch. d'Ecogia, 1290 Versoix, Switzerland \\ 2 Observatoire de Genève, 51 Ch. des Maillettes, 1290 Sauverny, Switzerland \\ ${ }^{3}$ Laboratoire d'Astrophysique de Grenoble, BP 43, 38041 Grenoble Cedex 9, France \\ 4 NASA Goddard Space Flight Center, Code 661, Greenbelt, MD 20771, USA \\ 5 Joint Center for Astrophysics, Department of Physics, University of Maryland, Baltimore County, Baltimore, \\ MD 21250, USA
}

Received 24 October 2003 / Accepted 4 March 2004

\begin{abstract}
We present the first BeppoSAX observation (0.1 to $220 \mathrm{keV})$ of the quasar Mrk 205. We have searched for the unusual Fe line profile observed in the XMM-Newton spectrum which has been widely discussed in the recent literature. We find no evidence for a broad, ionized Fe line component in our data. We detect for the first time a Compton hump in this object. When this component is included in the fit, the line strength diminishes, in agreement with a recent re-analysis of the XMM-Newton data, but with better constraints on the reflection component thanks to the PDS instrument (15-220 keV). We interpret this fact as another indication for illumination of distant, cold material rather than reprocessing in the highly ionized inner part of an accretion disk. We cannot constrain the presence of a high energy cutoff but we confirm the existence of a variable soft excess (one year time scale).
\end{abstract}

Key words. galaxies: active - galaxies: quasars: individual: Mrk 205 - X-rays: galaxies

\section{Introduction}

Discovered by Weedman (1970), the quasar Mrk 205 is seen through the southern spiral arm of the nearby spiral, barred galaxy NGC 4319 at a projected distance of roughly $40^{\prime \prime}$ from its center. From its radio luminosity at $6 \mathrm{~cm}$, Mrk 205 is classified as a radio-quiet quasar (Rush et al. 1996a), but it is still not clear whether it belongs to the radio-quiet quasar class (e.g., Bahcall et al. 1992) or to the Seyfert 1 class (e.g., Veron-Cetty $\&$ Veron 2001). Mrk 205 has a redshift $z=0.07085$ (Huchra et al. 1990) while NGC 4319 has $z=0.00468$ (Bowen et al. 1991).

In the standard paradigm of (radio-quiet) Active Galactic Nuclei (AGN), X-ray photons result from Compton upscattering of optical-UV photons in a hot thermal corona above the accretion disk surrounding a supermassive black hole. A large fraction of the seed photons are thought to be produced by thermal emission of the accretion disk itself, but Mrk 205 was found by McDowell et al. (1989) to have a weak blue bump. One of the possible scenarios which could explain this spectral morphology is that the bump may be highly variable and thus observed in a particularly weak state. In the thermal Comptonisation framework, blue bump variability would also induce hard X-ray variations.

A soft X-ray excess component may be interpreted as the hard tail of the blue bump (Walter \& Fink 1993;

Send offprint requests to: P. Favre,

e-mail: pascal.favre@obs.unige.ch
Brunner et al. 1997). So far, evidence for a soft excess in Mrk 205 was only reported in two observations; with EINSTEIN IPC (Wilkes \& Elvis 1987) and XMM-Newton (Reeves et al. 2001).

BeppoSAX, with its unique broad-band capabilities, allows us to extend the observation of Mrk 205 for the first time above $20 \mathrm{keV}$ to study the properties of the hard X-ray emission as well as of a potential reflection component. Indeed, some of the X-ray photons can be reprocessed in the surroundings, in particular in the disk itself giving rise to the neutral (or low-ionization) $\mathrm{Fe} \mathrm{K} \alpha$ line. While it is a common feature of Seyfert 1 galaxies (Nandra \& Pounds 1994), only a few quasars present a clear detection of such a component (Williams et al. 1992; Nandra et al. 1997). Furthermore, the centroid of the line was often measured at energies close to $6.7 \mathrm{keV}$, suggesting that its origin may be in an ionized layer of the accretion disk (Reeves \& Turner 2000), the neutral $\mathrm{Fe} \mathrm{K} \alpha$ being emitted in distant and cold material lying outside the broad line region. Reeves et al. (2001), who analysed the XMM-Newton data of Mrk 205, claimed the existence of a broad ionized component in addition to the neutral one in this object. However, Page et al. (2003) reprocessed the data including a reflection hump component in the fit and found little evidence for a broad, ionised component in the data.

In this paper, we present the analysis of three BeppoSAX observations of Mrk 205 for a total of $200 \mathrm{ks}$ of exposure time. In Sect. 2, we summarize the knowledge we had on Mrk 205 prior to our BeppoSAX observation. In Sect. 3, 
Table 1. Summary of the X-ray observations of Mrk 205; spectral parameters. The 2-10 keV flux was extrapolated from the best fit parameters.

\begin{tabular}{|c|c|c|c|c|}
\hline Mission, reference & Date & $\Gamma$ & $N_{\mathrm{H}}$ & Flux $_{2-10 \mathrm{keV}}$ \\
\hline & & & $10^{20} \mathrm{~cm}^{-2}$ & $10^{-11} \mathrm{erg} \mathrm{cm}^{-2} \mathrm{~s}^{-1}$ \\
\hline $\operatorname{EINSTEIN~(HRI),[\mathrm {a}]}$ & 22-11-1978 & $1.50^{f}$ & $3.00_{-0.10}^{+0.10}$ & $1.36_{-0.07}^{+0.07}$ \\
\hline $\operatorname{EINSTEIN}(I P C)^{\dagger},[\mathrm{b}]$ & 20-04-1980 & $1.80_{-0.20}^{+0.20}$ & $2.00_{-0.80}^{+0.90}$ & $1.18_{-0.05}^{+0.07}$ \\
\hline$E X O S A T^{\star},[\mathrm{c}]$ & $10-11-1983$ & $1.73_{-0.55}^{+0.62}$ & $1.33_{-1.30}^{+5.20}$ & $0.72_{-0.11}^{+0.10}$ \\
\hline$E X O S A T^{\star},[\mathrm{c}]$ & 27-01-1984 & $1.94_{-0.41}^{+0.74}$ & $2.84_{-2.35}^{+13.70}$ & $0.66_{-0.09}^{+0.08}$ \\
\hline$G I N G A^{\ddagger},[\mathrm{d}]$ & $16-01-1988$ & $1.95_{-0.11}^{+0.13}$ & $3.40_{-0}^{+3.40}$ & $1.01_{-0.10}^{+0.10}$ \\
\hline $\operatorname{ROSAT}(P S P C),[\mathrm{e}]$ & 10-11-1991 & $2.38_{-0.08}^{+0.08}$ & $3.24_{-0.24}^{+0.24}$ & $0.16_{-0.01}^{+0.01}$ \\
\hline ROSAT (PSPC), [e] & 15-04-1992 & $2.32_{-0.10}^{+0.10}$ & $3.32_{-0.30}^{+0.30}$ & $0.11_{-0.01}^{+0.01}$ \\
\hline$A S C A^{\ddagger},[\mathrm{f}]$ & 03-12-1994 & $2.13_{-0.03}^{+0.03}$ & $2.50_{-0.90}^{+0.90}$ & $0.75_{-0.09}^{+0.09}$ \\
\hline BeppoSAX, this work & 12-03-1997 & $1.77_{-0.06}^{+0.07}$ & $2.90_{-0}^{+1.18}$ & $1.21_{-0.02}^{+0.02}$ \\
\hline XMM-Newton ${ }^{\dagger \dagger},[\mathrm{g}]$ & $06-05-2000$ & $1.86_{-0.02}^{+0.02}$ & 4.30 & $0.47_{-0.05}^{+0.05}$ \\
\hline BeppoSA $X^{\dagger \neq}$, this work & $21-12-2001$ & $1.87_{-0.03}^{+0.02}$ & $2.90_{-0}^{+0.25}$ & $1.11_{-0.01}^{+0.01}$ \\
\hline BeppoSAX, this work & 05-01-2002 & $1.72_{-0.07}^{+0.07}$ & $3.69_{-0.79}^{+2.75}$ & $0.72_{-0.01}^{+0.01}$ \\
\hline
\end{tabular}

Note: Galactic $N_{\mathrm{H}}=2.90 \times 10^{20} \mathrm{~cm}^{-2}$ (Dickey \& Lockman 1990).

${ }^{\dagger}$ Evidence for a soft excess component reported.

^ Broken power law model preferred.

$\stackrel{\$}{\ddagger}$ Evidence for an $\mathrm{Fe} \mathrm{K} \alpha$ line reported.

${ }^{f}$ Fixed parameter.

we describe the data and their analysis while in Sect. 4 we compare our results and discuss them in the framework of the unified model.

\section{Previous X-ray observations of Mrk 205}

Mrk 205 was observed by EINSTEIN HRI (Tananbaum et al. 1979: [a]; Zamorani et al. 1981), EINSTEIN IPC (Wilkes \& Elvis 1987: [b]), EINSTEIN A-2 (Della Ceca et al. 1990), EXOSAT (Singh et al. 1991: [c]), GINGA (Williams et al. 1992: [d]), ASCA (Reeves \& Turner 2000: [f]), ROSAT PSPC (Fiore et al. 1994: [e]; Fiore 1994; Rush et al. 1996b; Ciliegi \& Maccacaro 1996; Fiore et al. 1997), ROSAT HRI (Arp 1996), XMM-Newton (Reeves et al. 2001: [g]; Page et al. 2003) and BeppoSAX (this work).

Mrk 205 has not been observed by RXTE nor Chandra to date.

We summarize the spectral parameters found in Fig. 1 and Table 1 . The spectral variability was important over the 24 years spanned by the data (Fig. 1, upper panel). The photon indexes were measured mostly over the range $2-10 \mathrm{keV}$ and thus were not affected by a soft excess component. However, the two ROSAT points $(1991,1992)$ are extrapolated values from the $0.11-2.45 \mathrm{keV}$, i.e. a spectral range very sensitive to the presence of a soft excess. These points are certainly overestimated. In addition, several papers discuss the ROSAT PSPC calibration problems which result in a measure of the AGN X-ray spectral slopes significantly steeper than those from other missions (Beckmann et al. 2002, and references therein).

We will discuss further in Sect. 4 the possible origin of the observed spectral variability in this object.

Little variation of the hydrogen column density were observed during the same period (Fig. 1, second panel from

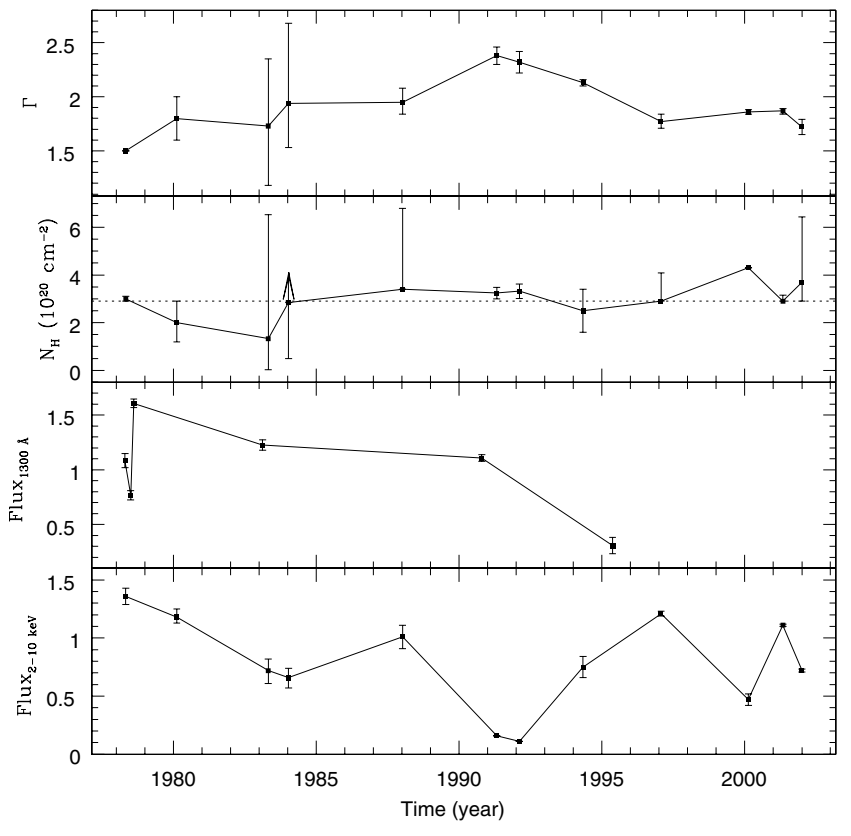

Fig. 1. Summary of the X-ray observations of Mrk 205; evolution with time of the photon index $\Gamma$, the hydrogen column density in the line of sight $N_{\mathrm{H}}$ (the dotted line denotes the Galactic value) and the $2-10 \mathrm{keV}$ flux (units: $10^{-11} \mathrm{erg} \mathrm{cm}^{-2} \mathrm{~s}^{-1}$ ). Data from Table 1. We have included as well an IUE light curve at $1300 \AA$ (units: $10^{-14} \mathrm{erg} \mathrm{cm}^{-2} \mathrm{~s}^{-1} \AA^{-1}$ ).

the top). An excess of absorption may be present in the XMM-Newton 2000 observation, its reality will be discussed in Sect. 3.7.

The $2-10 \mathrm{keV}$ flux seems to have decreased during the first $\sim 18$ years of observation, a trend possibly correlated with a similar trend seen in the $1300 \AA$ IUE light curve (Fig. 1, third panel from the top). Here again, the two ROSAT estimates of 
the $2-10 \mathrm{keV}$ flux may be underestimated due to the overestimation of the $2-10 \mathrm{keV}$ photon index.

In the remainder of this section, we will focus on the $X M M$-Newton observation that took place 18 months before our BeppoSAX pointings.

\subsection{XMM-Newton observation}

Reeves et al. (2001) reported an observation done with $X M M-N e w t o n$ on 6 May 2000. They measured a photon in$\operatorname{dex} \Gamma=1.86_{-0.02}^{+0.02}$ with an absorbing column in excess of the Galactic one limited to $N_{\mathrm{H}}<1.5 \times 10^{20} \mathrm{~cm}^{-2}$. Fitting the MOS data only they found evidence for an ionized Fe line component at $6.7 \mathrm{keV}$ along with the neutral $\mathrm{Fe} \mathrm{K} \alpha$ component. The same result was obtained using only the PN data, and thus the simultaneous fit of the data from both instruments gave tight constraints. They finally obtained the best fit with a narrow line resulting from neutral $\mathrm{Fe} \mathrm{K} \alpha$ emission at $6.39 \pm 0.02 \mathrm{keV}$ $(E W=56 \pm 23 \mathrm{eV})$ and a broad component at $6.74 \pm 0.12 \mathrm{keV}$ $\left(E W=135_{-60}^{+65} \mathrm{eV}\right)$. They did not detect an Fe $\mathrm{K}$ edge nor a reflection component but claimed that they could not rule out their presence.

The observations were consistent with a relativisticallybroadened accretion disk line model (Fabian et al. 1989) only if the disk ionization was high enough to produce He-like Fe at $6.7 \mathrm{keV}$ and the neutral component was present.

A clear soft excess was also detected, but fitting it with a black body gave a much too high temperature $(k T=120 \pm$ $8 \mathrm{eV}$ ) to be direct emission from the accretion disk. An explanation is enhanced emission from the upper layers of the ionized accretion disk. The fact that the model of Compton reflection from ionized material (PEXRIV: Magdziarz \& Zdziarski 1995) gave good results (with $\xi=300 \pm 80 \mathrm{erg} \mathrm{cm} \mathrm{s}^{-1}$ ) was probably confirming this (see also Nayakshin et al. 2000). No warm absorber was found; the limits on the OVII and OVIII edges were $\tau<0.12$ and $\tau<0.15$ respectively. Page et al. (2003) reprocessed these data and found that an acceptable fit was obtained with a power law plus neutral emission line at $6.4 \mathrm{keV}$. They noticed that a better fit was obtained either with a Compton reflection component or with an ionized emission line, with a similar goodness of fit. The presence of ionized relativistic material was thus not unambiguously detected in this object, the illumination of a distant cold material providing a simpler self-consistent explanation.

\section{BeppoSAX observation}

Mrk 205 was observed by BeppoSAX (Boella et al. 1997a) on 21 December 2001 (LECS: 47582 s, MECS: 165458 s, PDS: 70549 s) and on 5 January 2002 (LECS: $3611 \mathrm{~s}$, MECS: 32603 s, PDS: 12606 s). In addition, public data from the archive (12 March 1997, LECS: 2525 s, MECS: 14727 s, PDS: $6646 \mathrm{~s}$; part of the core program and pointed at NGC 4319) are included in the present study.

LECS (Low Energy Concentrator Spectrometer, Parmar et al. 1997) and MECS (Medium Energy Concentrator Spectrometer, Boella et al. 1997b) data were reduced following the standard procedures. For PDS (Phoswich Detector
System, Frontera et al. 1997) data, high level products from the Sax Data Center were used. LECS and MECS data have been screened according to Fiore et al. (1999) to produce equalized event files using an extraction radius of $8^{\prime}$ for LECS and $4^{\prime}$ for MECS. Data from the two MECS units have been merged to increase the signal-to-noise ratio.

For the longest exposure (2001), the average source flux in the $2-10 \mathrm{keV}$ band (MECS data only, power law model with Galactic absorption) was $(1.11 \pm 0.01) \times 10^{-11} \mathrm{erg} \mathrm{cm}^{-2} \mathrm{~s}^{-1}$ corresponding to a luminosity $L=2.55 \times 10^{44} \mathrm{erg} \mathrm{s}^{-1}$ in the 2-10 keV band rest frame (here and in the following $H_{0}=$ $50 \mathrm{~km} \mathrm{~s}^{-1} \mathrm{Mpc}^{-1}$ ). From that luminosity, a black hole mass of about $2 \times 10^{6} M_{\odot} L_{\text {Edd }} / L$ could be inferred. These values are more typical of a luminous Seyfert 1 galaxy than a quasar.

The $2-10 \mathrm{keV}$ flux measured in 2001 was twice the value measured 18 months before by XMM-Newton (Fig. 1).

\subsection{Image analysis: Unlikely influence from NGC 4319 and other sources}

We analysed the MECS 1.3-10 keV image (angular resolution 42") of the 2001 observation and compared it with a ROSAT HRI 0.1-2.4 keV image (angular resolution of 5"). For the HRI image, Arp (1996) evaluated the shift of the position from the optical to X-ray to be of at most 7", equivalent to one MECS pixel. Three sources were identified in the MECS image, Mrk 205 included. Being in the field of view of the PDS also (1.4 degrees while the MECS field of view is $28^{\prime}$ ), we investigated if one of the other two sources could contribute to the Mrk 205 PDS spectra.

In addition, we had to make sure that NGC 4319 located at 40" from Mrk 205 (and thus not resolved by this instrument) was not emitting in the X-rays (we used the following J2000 coordinates for Mrk 205: $\alpha=12^{\mathrm{h}} 21^{\mathrm{m}} 44.12^{\mathrm{s}}$ $\delta=+75^{\circ} 18^{\prime} 38.2^{\prime \prime}$ and for NGC 4319: $\alpha=12^{\mathrm{h}} 21^{\mathrm{m}} 44.07^{\mathrm{s}}$ $\delta=+75^{\circ} 19^{\prime} 21.3^{\prime \prime}$, these are indicated by circles in Fig. 2). We discuss these points below.

1. According to the profile displayed in Fig. 2 (right panel), NGC 4319 is located in a zone in which half of the MECS peak count rate is received. Furthermore the shape itself does not allow us to tell if NGC 4319 is really detected (the break in the profile can be a feature of the MECS PSF). It was not detected by ROSAT HRI (Arp 1996) in the soft $\mathrm{X}$-ray domain, but it could be Compton thick with emission only detected above $5-10 \mathrm{keV}$. However, there are no radio sources (apart from Mrk 205) above $2.5 \mathrm{mJy}$ within a radius of $1.5^{\prime}$ of Mrk 205 in the NVSS catalog (Condon et al. 1998). Thus it is very unlikely that NGC 4319 is an AGN.

2. We have fitted the 2001 MECS spectrum with a power law, extrapolated the model in the PDS domain and added the PDS data without fitting them but including an intercalibration constant MECS/PDS frozen to the recommended value of 0.88 (see Fiore et al. 1999). This does not take into account any reflection component. Figure 3 shows the resulting spectra along with the residuals of both data sets with respect to the MECS best fit model. It is very unlikely that emission from NGC 4319 contributes to the excess 


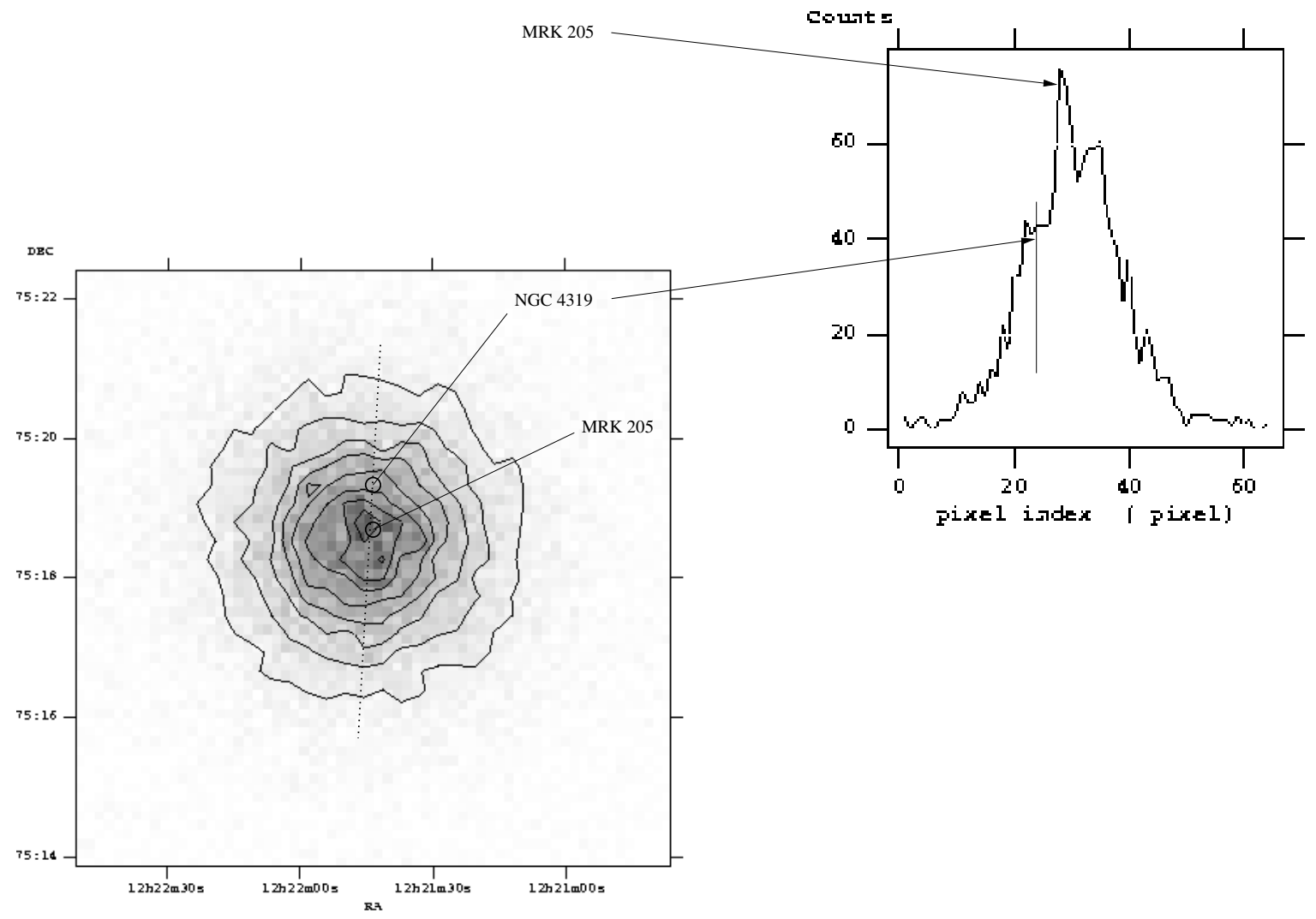

Fig. 2. Left panel: MECS intensity image of the 2001 observation with contours at (from the outside) 7.7, 15.5, 23.3, 31.1, 38.8, 46.6, 54.4, 62.2 counts. Right panel: profile along the dotted line displayed on the left panel (1 MECS pixel is 7"). NGC 4319 is located 6 pixels on the left of the central peak in a region which accounts for $54 \%$ of the peak value.

at $20 \mathrm{keV}$ and matches the extrapolation of the MECS flux at high energies.

3. The other sources in the MECS data are XMMU J122206.4 $+752613^{1}$ which is a narrow emission line galaxy and NGC 4291, a non-active elliptical galaxy. The first has a MECS count rate 10 times smaller than Mrk 205 while for the latter it is 100 times smaller. According to Fiore et al. (1999), those sources are certainly in the residual PDS count rate (estimated by comparing to the known MECS count rate) obtained in blank fields (Guainazzi \& Matteuzzi 1997).

From these different arguments, we conclude that neither NGC 4319 nor one of the two other sources detected by MECS contribute significantly to the X-ray spectrum of Mrk 205.

\subsection{Possible absorption in the spiral arms of NGC 4319}

NGC 4319 is an extremely disrupted spiral galaxy of type $S_{\text {pec }}$ (Arp 1996) with an ISM very different from the Milky Way's ISM. The ISM of NGC 4319 has been thoroughly studied by various authors who have found $\mathrm{Ca}$ II $\mathrm{K}$ and $\mathrm{Mg} \mathrm{I} \mathrm{b}$ absorption features at the redshift of NGC 4319 (Stockton et al. 1979).

\footnotetext{
1 The source XMMU J122206.4+752613 corresponds to MS 1219.9+7542 and to ROSAT 1RXS J122214.2+752624.
}

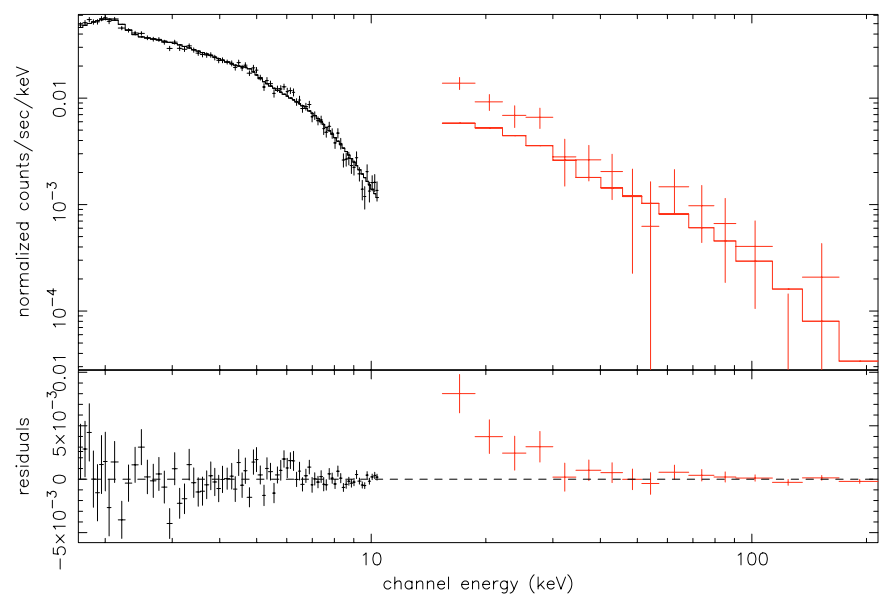

Fig. 3. Upper panel: fit of the 2001 MECS data with a power law and absorption frozen to the Galactic value. PDS data are included without being fitted but with an intercalibration constant frozen to 0.88. Lower panel: residuals with respect to the MECS best fit model.

Bahcall et al. (1992) succeeded in the detection of the Mg II doublet in NGC 4319 and deduced an approximative $\mathrm{H}$ I column density of about $3 \times 10^{18} \mathrm{~cm}^{-2}$ from the Mg II column density, precised by Womble et al. (1993) to be $N(\mathrm{HI})<$ $5.2 \times 10^{19} \mathrm{~cm}^{-2}$.

The gas in the inter-arm region of NGC 4319 shows a higher grade of ionisation than in the Milky Way and the 

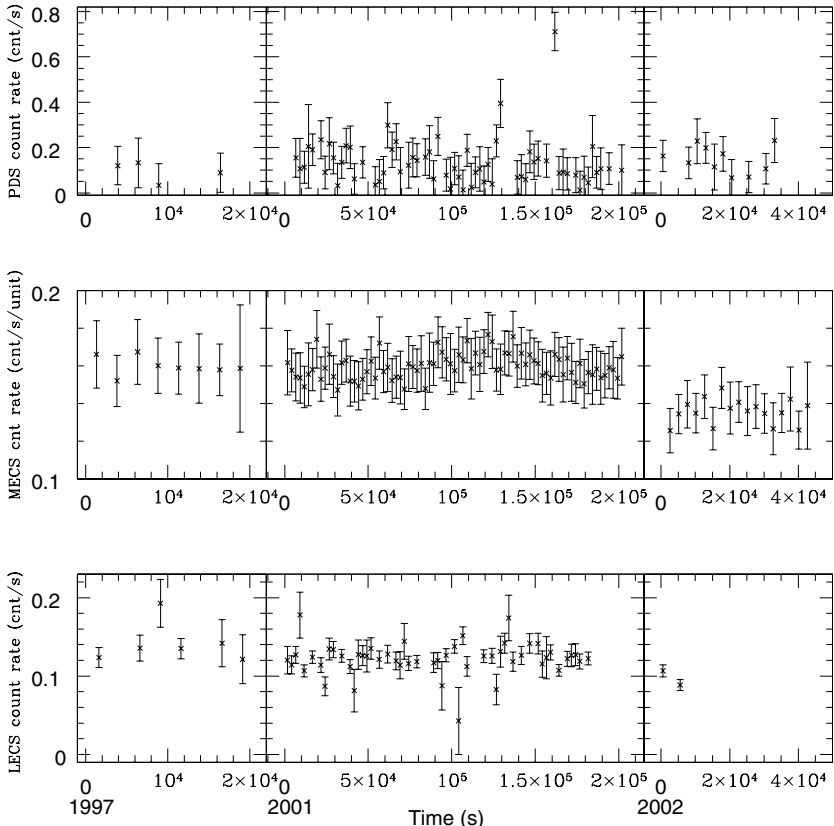

Fig. 4. Light curves of the 3 observations. Top panels: PDS 13-200 keV, middle panels: MECS 2-10 keV (count rate per MECS unit), bottom panels: LECS 0.01-2 keV.

unusually low amount of $\mathrm{HI}$ (or Mg II) is not understood for this kind of late-type galaxy.

The X-ray absorption in the $0.5-2.0 \mathrm{keV}$ energy range is however dominated by $\mathrm{CNO} \mathrm{K}$-shell absorption, but no evidence for an enhanced abundance of heavy elements is reported in the literature. Thus, we do not find evidence for absorption in the Mrk 205 data due to the presence of NGC 4319 in the line of sight.

\subsection{Variability}

We produced light-curves from the event files using Xronos 5.16 in a single binning of $2500 \mathrm{~s}$ to ease intercomparison between the different observations (Fig. 4). Note that 15 days separate the middle panel of Fig. 4 from the right panel. LECS and MECS light curves of the statistically best observation (2001) were surprisingly different. Cross-correlation analysis gave a weak correlation coefficient between these two light curves.

From 1997 to 2001, the LECS and MECS count rates remained constant. Since a MECS unit was lost between those 2 observations, we have plotted in Fig. 4 the MECS light curves for a single unit. The average PDS count rate seems to have increased by about $40 \%$, but this value is very inaccurate due to the few points in 1997.

From 2001 to 2002, the LECS average count rate seemed to have dropped by about $16 \%$ percent, but this value is based on a small fraction of the observation time. The MECS average count rate dropped by $15 \%$, while the PDS count rate remained constant.

From the December 2001 observation (Fig. 4, middle panels; see also Fig. 5) one sees that no significant variability was observed on very short time scales (of the order of hours).

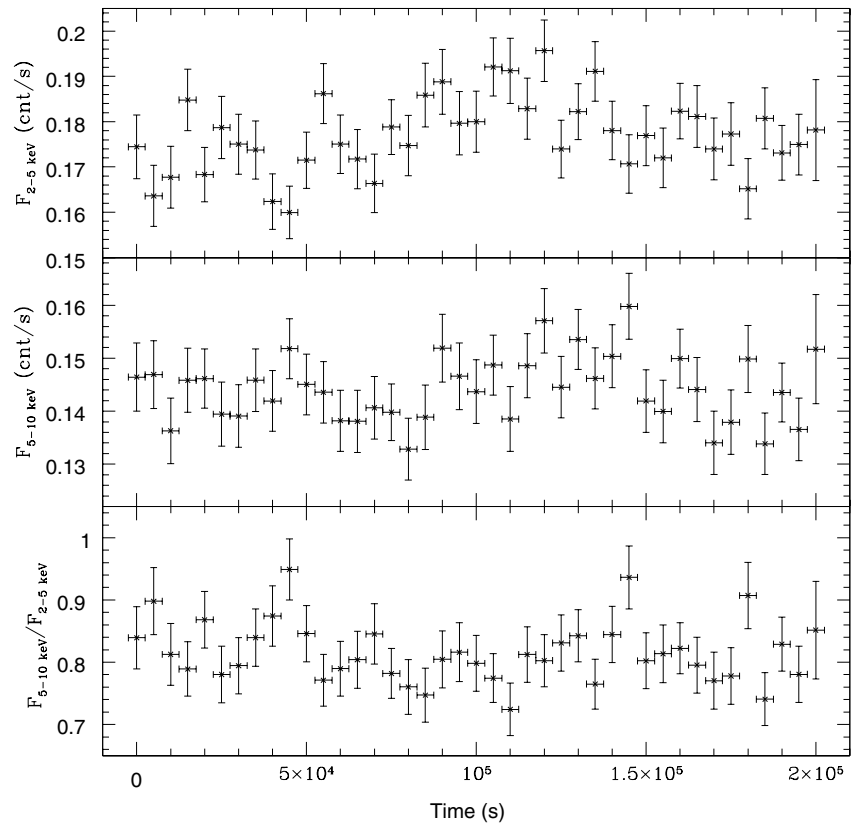

Fig. 5. Observation of December 2001, MECS data. Top panel: 2-5 keV light curve, middle panel: 5-10 keV light curve, bottom panel: hardness ratio.

On the other hand, long-term variability (on week and year time scales) was quite significant (Fig. 1).

In Fig. 5, we display the light curves 2-5 keV and 5-10 keV along with the hardness ratio for the longest observation (2001). The $2-5 \mathrm{keV}$ energy band showed a weak minimum ( $10 \%$ below the average flux) around $4 \times 10^{4} \mathrm{~s}$ which was not present in the 5-10 keV range. The resulting hardness ratio thus showed a hardening around that time. The differences in the two light curves are certainly not due to $N_{\mathrm{H}}$ variations as a value of the order of $10^{20} \mathrm{~cm}^{-2}$ is unlikely to change the spectra above $2 \mathrm{keV}$.

\subsection{Model independent spectral analysis}

To study the spectral variations between the different observations, we divided the 3 spectra (LECS, MECS, PDS) of the 2001 observation from the 3 spectra of the 2002 observation (Fig. 6). We also divided the 3 spectra of the 2001 observation from the 3 spectra of the 1997 observation (Fig. 7). We took the ratios for each energy bin. One sees an important variation in the 2-10 keV energy range between the 2001 and 2002 observation which did not occur between the 1997 and 2001 observation, in agreement with Fig. 4. A rough comparison of Figs. 6 and 7 shows that the LECS and PDS points may have been enhanced as well in Fig. 6, but the signal-to-noise ratio is not good enough to conclude that the soft $0.1-2.0 \mathrm{keV}$ or hard $10-220 \mathrm{keV}$ bands varied significantly.

\subsection{Spectral analysis}

The spectral fitting was performed with XSPEC 11.2.0. In the following, all errors are quoted at the $90 \%$ confidence level for one interesting parameter. We have used the recommended 


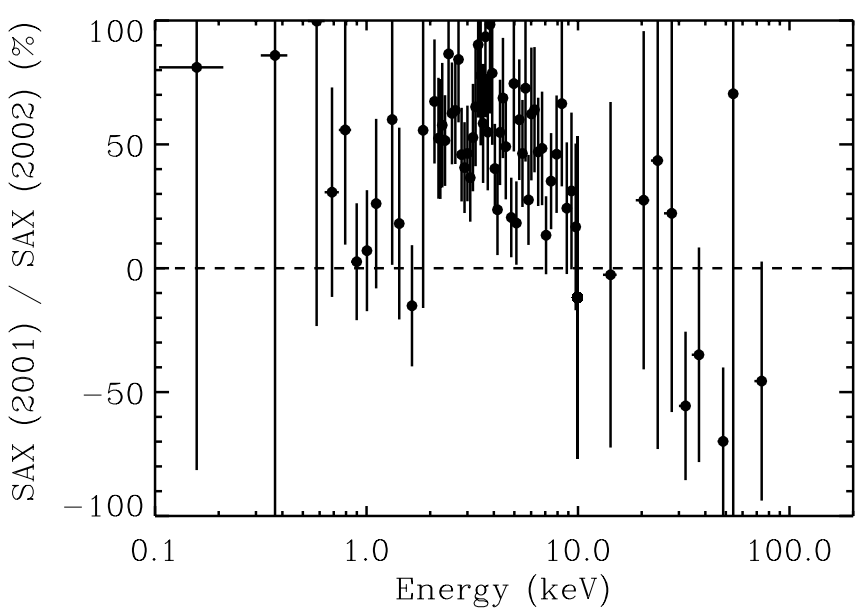

Fig. 6. Spectral variations between 2001 and 2002 (ratio of the 2001 spectra to the 2002 spectra).

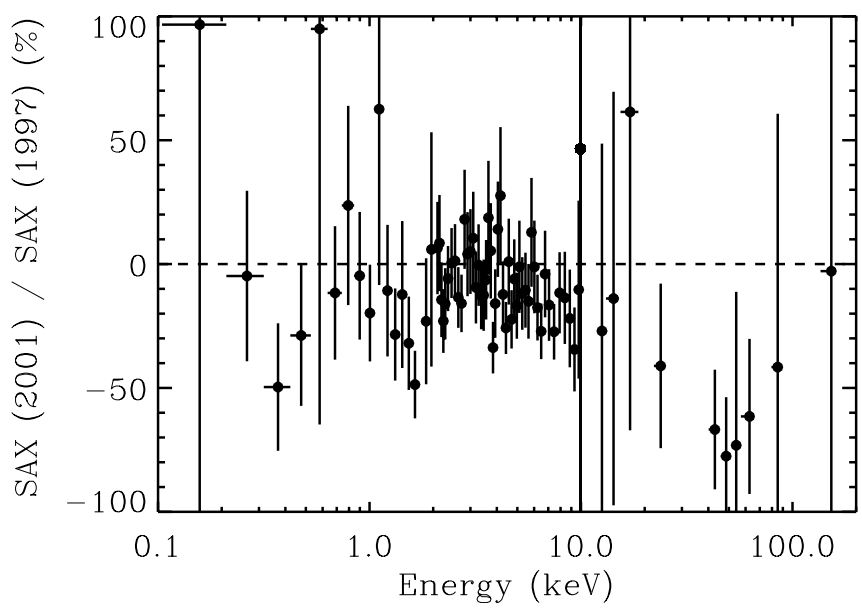

Fig. 7. Spectral variations between 1997 and 2001 (ratio of the 2001 spectra to the 1997 spectra).

energy bounds (LECS: $0.12-4.0 \mathrm{keV}$, MECS: $1.65-10.5 \mathrm{keV}$, PDS: $15-220 \mathrm{keV}$ ), and the intercalibration "constants" were free to vary in the intervals $0.7-1.0$ and $0.82-0.90$ for LECS/MECS, PDS/MECS respectively (Fiore et al. 1999).

The upper panel of Fig. 8 represents a fit of the 2001 data with a power law model modified by interstellar photoelectric absorption. The photoelectric absorption cross-sections used were those of Morrison \& McCammon (1983).

The lower panels of Fig. 8 show the fits residuals with the same model for the 1997 and 2002 observations. The best fit spectral parameters found are presented in Table 2.

The fit of the 2001 observation is poor with an excess in the $15-40 \mathrm{keV}$ range as well as in the soft X-ray, up to about $1 \mathrm{keV}$. Finally a small feature around $6 \mathrm{keV}$ is present.

For the 1997 and 2002 observations, the fits are already acceptable with this simple model for the continuum. In the 2002 observation, a line-like feature is clearly detected around $1 \mathrm{keV}$ and a small excess in the range $15-40 \mathrm{keV}$ may be present. The signal-to-noise ratio of the continuum is not good enough to accurately constrain more complex physical models.
Table 2. Fits of the BeppoSAX data using a power law plus Galactic absorption model: best fit spectral parameters.

\begin{tabular}{lccc}
\hline \hline Observation & $\Gamma$ & $N_{\mathrm{H}}$ & $\chi^{2}$ (d.o.f.), proba. \\
\hline \multicolumn{4}{c}{$10^{20} \mathrm{~cm}^{-2}$} \\
\hline 2001 & $1.87_{-0.03}^{+0.02}$ & $<3.15^{p}$ & $182.23(139), 0.008$ \\
1997 & $1.77_{-0.06}^{+0.07}$ & $<4.08^{p}$ & $106.88(99), 0.277$ \\
2002 & $1.72_{-0.07}^{+0.07}$ & $3.69_{-0.79}^{+2.75}$ & $104.70(99), 0.328$ \\
\hline
\end{tabular}

${ }^{p}$ Pegged at Galactic value $N_{\mathrm{H}}=2.90 \times 10^{20} \mathrm{~cm}^{-2}$.

\subsection{Spectral analysis: Reflection component}

We started a more detailed analysis by fitting the 2001 observation data above $\sim 2 \mathrm{keV}$, i.e. using MECS and PDS data only. As $N_{\mathrm{H}}$ was not constrained in this case, we fixed it to the Galactic value. We tried 2 different models for the continuum shape.

The first was a cut-off power law plus a Compton reflection from dense, neutral matter (PEXRAV: Magdziarz \& Zdziarski 1995). This model is meant to describe the effects of Compton scattering of photons from an X-ray source associated with the inner parts of the accretion disk. The spectrum of reflected $\mathrm{X}$-rays was computed as a function of disk inclination using the transfer function of Magdziarz \& Zdziarski (1995). The free parameters of this model are the spectral index $\Gamma$ of the primary power law, the folding (upper cutoff) energy $E_{\mathrm{c}}$ of the primary X-ray spectrum and the normalization of the reflection component $R$. In our fits, the inclination angle of the disk was frozen to 30 degrees as is usually assumed for Seyfert 1 galaxies, while the abundances of $\mathrm{Fe}$ and other heavy elements were fixed to the solar values.

The second model was a more physical model of thermal Comptonisation for a disk-corona configuration in a slab geometry (COMPHA: Haardt 1993; Haardt et al. 1997). The table model for XSPEC ${ }^{2}$ includes both the primary continuum and the reflection. The reflection component was computed following White et al. (1988) and Lightman \& White (1988) assuming reflection off neutral matter and a constant spectral shape (angle-averaged) for the reflected photons. The free parameters of the model are the corona temperature normalized to the electron mass $k T_{\mathrm{e}} / m_{\mathrm{e}} c^{2}$, the corona optical depth $\tau$ and the normalization of the reflection component $R$ (without the Comptonisation effects). In our fits, the temperature $k T_{\mathrm{bb}}$ of the disk (which is assumed to radiate like a single black-body) was frozen to $10 \mathrm{eV}$ while the inclination angle of the disk was frozen to 30 degrees.

Both continuum models were completed by the addition of a Gaussian emission line to model the $\mathrm{Fe} \mathrm{K} \alpha$ line with a width of $0.1 \mathrm{keV}$ frozen (a more detailed analysis on the Fe line parameters is made below). The best fit spectral parameters are given in Table 3.

The fit of the observation made in 2001 is improved by using PEXRAV without a cutoff $\left(\Delta \chi^{2}=29.51\right.$ for 1 more parameters; F-test value 25.20 and probability $\left.2.57 \times 10^{-6}\right)$. It is slightly improved when including the cutoff $\left(\Delta \chi^{2}=1.97\right.$ for 1 more parameters; F-test value 1.70 and probability 0.196 ) and

\footnotetext{
${ }^{2}$ http://pitto.mib.infn.it/ haardt/ATABLES
} 


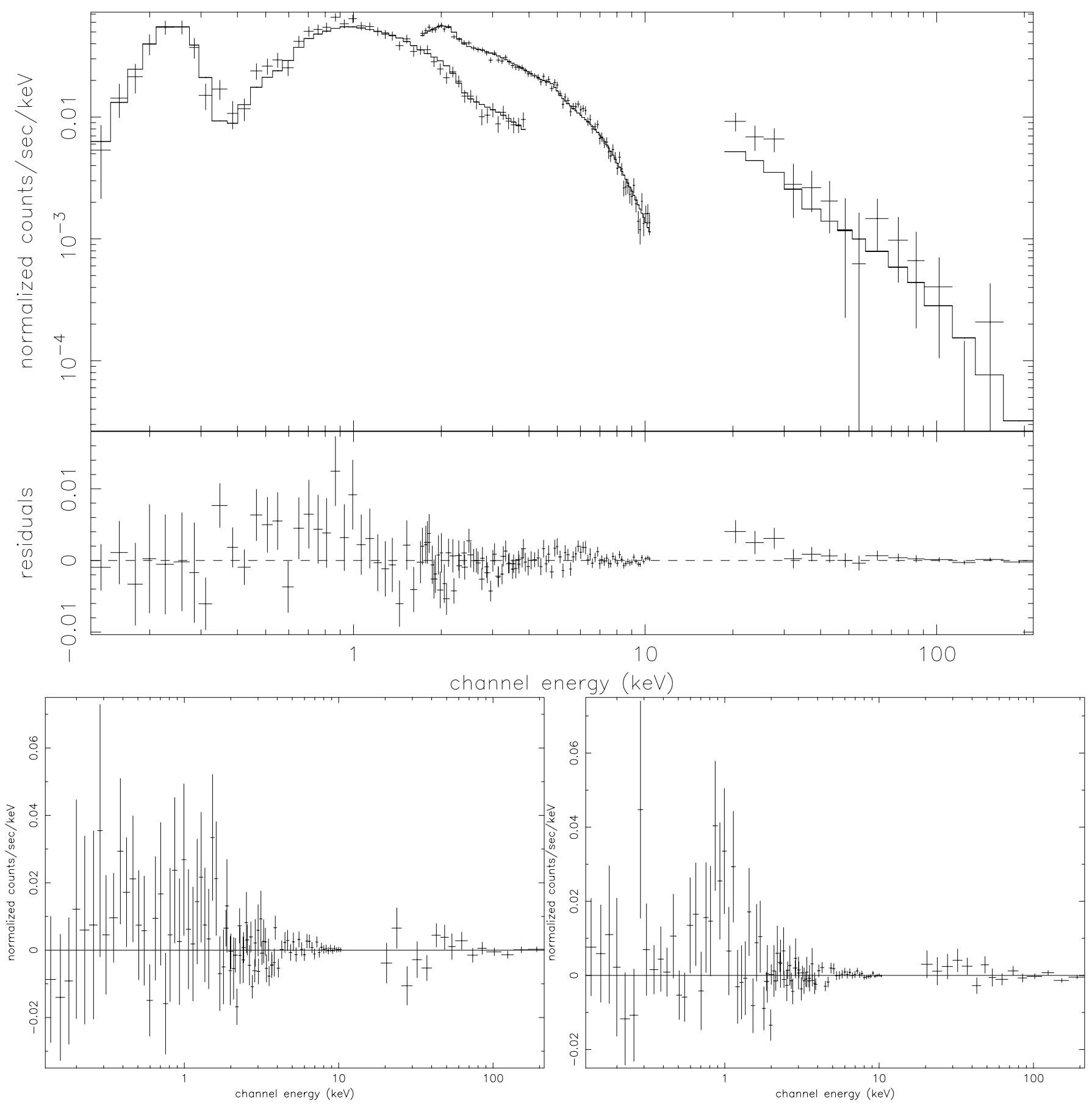

Fig. 8. Upper panel: broad-band (folded) spectrum of the 2001 observation. The spectrum is fitted with a power law plus Galactic absorption model. Lower panel, left side: residuals after the fit of the 1997 observation with the same model. Lower panel, right side: as above, for 2002.

the line $\left(\Delta \chi^{2}=5.60\right.$ for 2 more parameters; F-test value 2.48 and probability $8.93 \times 10^{-2}$ ). The solid angle subtended by the reflector is poorly constrained (Fig. 9). Figure 10 shows that the cutoff upper limit is not constrained.

The $\chi^{2}$ is not significantly improved by PEXRAV for the two other observations (Table 3 ). We thus cannot constrain the presence of this component in 1997 and 2002.

The fit of the December 2001 observation is improved by using COMPHA when compared to a power law $\left(\Delta \chi^{2}=30.11\right.$ for 2 more parameters; F-test value 12.80 and probability $1.30 \times 10^{-5}$ ). It is slightly improved by including the Fe line
$\left(\Delta \chi^{2}=7.17\right.$ for 2 more parameters; F-test value 3.19 and probability $\left.4.61 \times 10^{-2}\right)$.

Thus, fitting with the thermal Comptonisation model COMPHA does not improve the fit of the 2001 observation in comparison to the Compton reflection from cold material PEXRAV fits.

\subsection{Spectral analysis: Soft excess}

We now consider the broad-band 2001 spectrum, adding the LECS $0.12-4.0 \mathrm{keV}$ data to the MECS and PDS data. Freezing 
Table 3. Reflection component: fits of the MECS and PDS data (1.65-220 keV). A stands for photoelectric absorption, PL for power law, PEXRAV for the Compton reflection from cold material and COMPHA for the thermal Comptonisation model. $\Gamma$ is the photon index of the primary power law, $E_{\mathrm{c}}$ is the folding (upper cutoff) energy, $R$ is the normalization of the reflection component, $k T_{\mathrm{e}} / m_{\mathrm{e}} c^{2}$ is the corona temperature (normalized to the electron mass), $\tau$ is the corona optical depth, $E_{\mathrm{Fe}}$ is the $\mathrm{Fe} \mathrm{K} \alpha$ line energy (rest frame) and $E W_{\mathrm{Fe}}$ its equivalent width. As $N_{\mathrm{H}}$ is not constrained above $1.65 \mathrm{keV}$, we fixed it to the Galactic value. In addition, the results of a fit of the XMM-Newton data above $2 \mathrm{keV}$ are presented.

\begin{tabular}{|c|c|c|c|c|c|c|c|c|c|}
\hline Obs. & Model & $\Gamma$ & $E_{\mathrm{c}}$ & $R$ & $k T_{\mathrm{e}} / m_{\mathrm{e}} c^{2}$ & $\tau$ & $E_{\mathrm{Fe}}$ & $E W_{\mathrm{Fe}}$ & $\chi^{2}$ (d.o.f.), proba. \\
\hline & & & $\mathrm{keV}$ & & & & $\mathrm{keV}$ & $\mathrm{eV}$ & \\
\hline \multirow[t]{3}{*}{ SAX 2001} & $\mathrm{~A}+\mathrm{PL}$ & $1.83_{-0.03}^{+0.03}$ & - & - & - & - & - & - & 137.47(93), 0.002 \\
\hline & $\mathrm{A}+\mathrm{PEXRAV}+\mathrm{G}$ & $2.00_{-0.03}^{+0.03}$ & $487.17_{-304.40}^{+u}$ & $1.74_{-0.32}^{+0.25}$ & - & - & $6.51_{-0.55}^{+0.42}$ & 71.30 & $100.38(89), 0.192$ \\
\hline & $\mathrm{A}+\mathrm{COMPHA}+\mathrm{G}$ & - & - & $3.22_{-1.35}^{+0.99}$ & $0.32_{-u}^{+0.04}$ & $0.15_{-0.08}^{+0.33}$ & $6.52_{-0.18}^{+0.18}$ & 70.30 & $100.39(89), 0.192$ \\
\hline \multirow[t]{3}{*}{ SAX 1997} & $\mathrm{~A}+\mathrm{PL}$ & $1.74_{-0.08}^{+0.09}$ & - & - & - & - & - & - & $48.36(53), 0.655$ \\
\hline & $\mathrm{A}+\mathrm{PEXRAV}+\mathrm{G}$ & $1.77_{-0.13}^{+0.70}$ & $240.00^{f}$ & $<8.78$ & - & - & $6.40^{f}$ & $<1$ & $48.16(51), 0.587$ \\
\hline & $\mathrm{A}+\mathrm{COMPHA}+\mathrm{G}$ & - & - & $2.50^{f}$ & $0.48_{-u}^{+0.04}$ & $0.05_{-u}^{+u}$ & $6.40^{f}$ & $<1$ & 47.66(51), 0.607 \\
\hline \multirow[t]{3}{*}{ SAX 2002} & $\mathrm{~A}+\mathrm{PL}$ & $1.69_{-0.07}^{+0.07}$ & - & - & - & - & - & - & 49.91(53), 0.595 \\
\hline & $\mathrm{A}+\mathrm{PEXRAV}+\mathrm{G}$ & $1.80_{-0.16}^{+0.25}$ & $228.30_{-193.00}^{+u}$ & $<3.47$ & - & - & $6.40^{f}$ & 34.90 & $46.89(50), 0.599$ \\
\hline & $\mathrm{A}+\mathrm{COMPHA}+\mathrm{G}$ & - & - & $2.43_{-1.97}^{+4.41}$ & $0.37_{-0.09}^{+0.13}$ & $0.19_{-u}^{+u}$ & $6.40^{f}$ & 38.90 & $46.27(50), 0.624$ \\
\hline XMM 2000 & $\mathrm{~A}+\mathrm{PEXRAV}+\mathrm{G}$ & $1.73_{-0.14}^{+0.14}$ & $500.00^{f}$ & $0.48_{-0.48}^{+1.09}$ & - & - & $6.40^{f}$ & 75 & 75.24(54), 0.029 \\
\hline
\end{tabular}

${ }^{f}$ Parameters fixed in the fit.

${ }^{u}$ Unbounded parameters.

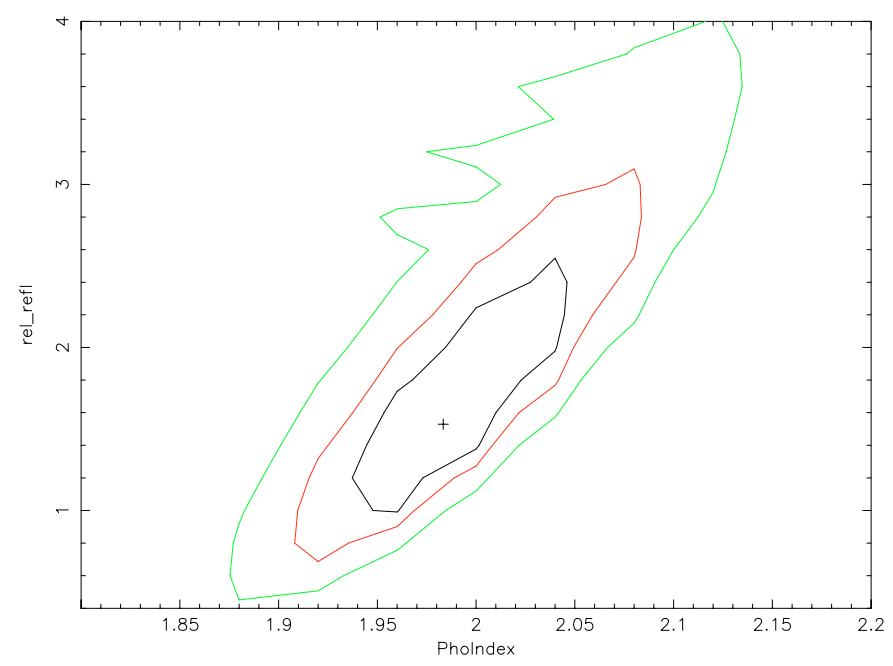

Fig. 9. Contour plot $R-\Gamma$ after the fit of the 2001 observation with the Compton reflection from cold material model plus an $\mathrm{Fe} \mathrm{K} \alpha$ line.

all the parameters of the best fit PEXRAV model above $2 \mathrm{keV}$ and fitting again the broad-band spectrum, we get an $N_{\mathrm{H}}=$ $\left(3.46_{-0.22}^{+0.26}\right) \times 10^{20} \mathrm{~cm}^{-2}$, and a $\chi^{2}$ of 138.49 for 142 degrees of freedom (d.o.f.). We found no clear evidence for a soft excess of photons in the LECS, even if the intercalibration constant is set to its lowest value of 0.7. To have an upper limit on a soft excess component, we add a black body to our model, fixing its temperature to the one obtained by Reeves et al. (2001) with the XMM-Newton data, i.e. $k T=120 \mathrm{eV}$. We found an $N_{\mathrm{H}}=\left(3.60_{-0.29}^{+0.36}\right) \times 10^{20} \mathrm{~cm}^{-2}$ and a $\Delta \chi^{2}=1.73$ for 1 more parameter; F-test value 1.78 and probability 0.184 . The soft excess here is much weaker ( $\sim 3 \%$ of the emission over the range

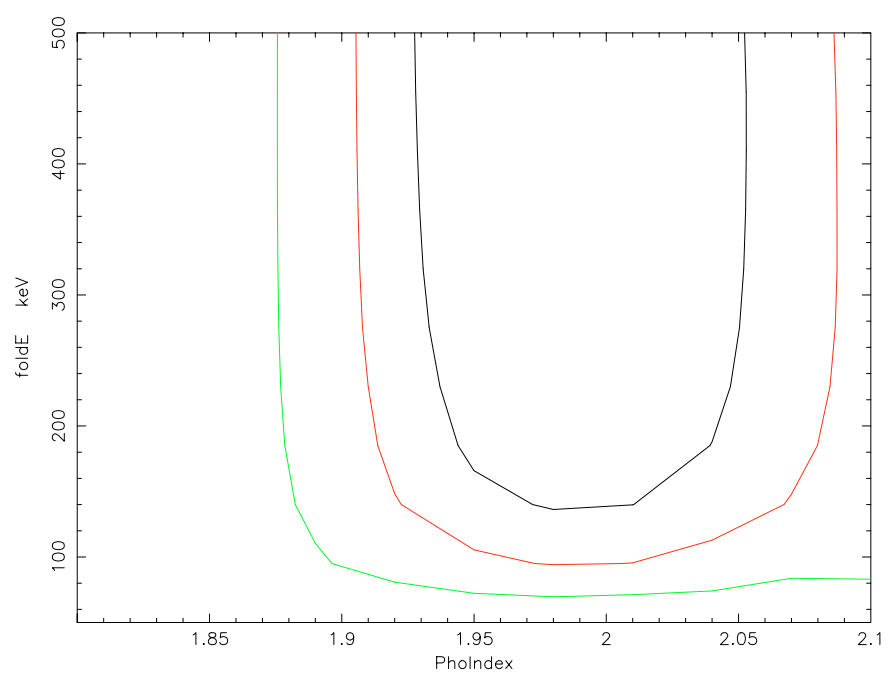

Fig. 10. Contour plot $E_{\mathrm{c}}-\Gamma$ after the fit of the 2001 observation with the Compton reflection from cold material model plus an $\mathrm{Fe} \mathrm{K} \alpha$ line.

$0.5-2.0 \mathrm{keV})$ than it was claimed in the XMM-Newton observation ( $\sim 15 \%$ of the emission in the range $0.5-2.0 \mathrm{keV})$.

We re-analysed the XMM-Newton PN data to see whether the soft excess component was as important as claimed by Reeves et al. (2001) when the reflection component was present. Indeed, Reeves et al. (2001) discussed the soft excess component modeling the continuum with a power law while Page et al. (2003) used the Compton reflection model but stopped their analysis at $3 \mathrm{keV}$. As before, we deduced the parameters of the continuum fitting the data above $2 \mathrm{keV}$ (Table 3 ). The PEXRAV model produced a $\chi^{2}$ of 75.24 (54 d.o.f.) with a realistic reflection parameter $R$ of 0.48 . We then fixed the continuum and included the 0.5 to $2.0 \mathrm{keV}$ points. The residuals showed 
Table 4. Soft excess: fits of the LECS, MECS, PDS data and comparison with XMM-Newton data. A stands for photoelectric absorption, PEXRAV for the Compton reflection from cold material, $G$ for a Gaussian line and BB for a black body. We first consider the spectra above $2 \mathrm{keV}$, freezing all the parameters of the continuum $\left(\Gamma, R, E_{\mathrm{c}}\right)$ to their best fit value of Table 3 . We then add the data below $2 \mathrm{keV}$, add a soft excess component, and fit again the broad-band spectrum.

\begin{tabular}{llcccc}
\hline \hline Obs. & Model & $N_{\mathrm{H}}$ & $k T_{\mathrm{BB}}$ & $\chi^{2}$ (d.o.f.) & Proba. \\
\hline & & $10^{20} \mathrm{~cm}^{-2}$ & $\mathrm{eV}$ & & \\
\hline BeppoSAX 2001 & A+PEXRAV+G+BB & $3.60_{-0.31}^{+0.50}$ & $100.00_{-99.90}^{+u}$ & $137.40(140)$ & 0.546 \\
\hline$X M M$ 2000 & A+PEXRAV+G+BB & $<3.14^{p}$ & $164.86_{-8.57}^{+4.85}$ & $325.09(231)$ & $<0.001$ \\
\hline & & & & \\
${ }^{u}$ Unbounded parameters. \\
${ }^{p}$ Pegged at Galactic value $N_{\mathrm{H}}=2.90 \times 10^{20} \mathrm{~cm}^{-2}$.
\end{tabular}

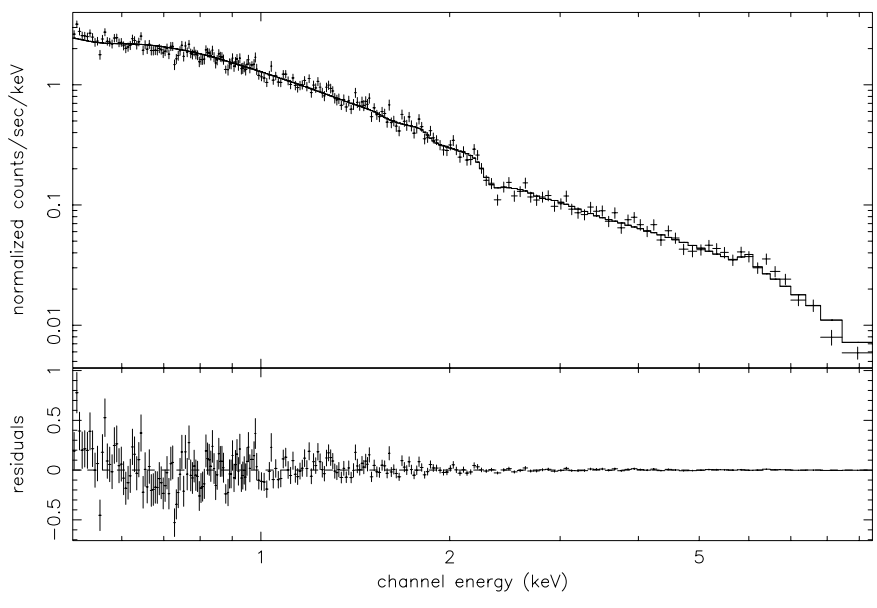

Fig. 11. XMM-Newton 2000 data, fit with the Compton reflection from cold material model.

a clear excess below $2 \mathrm{keV}$. Adding a black body with a temperature of $120 \mathrm{eV}$ did not produce a good fit $\left(\chi^{2}=463.29\right.$ for 232 d.o.f.) but, when this parameter was released, the fit was much better and the temperature significantly increased (see Table 4 and Fig. 11). We no longer found an unusual value of $N_{\mathrm{H}}$ (Fig. 1).

The contribution of the soft excess component is thus more important than previously claimed: it represents $\sim 23 \%$ of the emission in the range 0.5 to $2.0 \mathrm{keV}$.

However, the black body parameters do show suspicious values meaning that the emission is probably not a simple black body.

\subsection{Spectral analysis: $\mathrm{Fe} \mathrm{K} \alpha$ line}

To assess the importance of an $\mathrm{Fe} \mathrm{K} \alpha$ line in our data, we concentrate on the MECS data. In this case, $N_{\mathrm{H}}$ is not constrained, we thus fixed it to the Galactic value. Using only the power law model, it is clear from the residuals in the lower panel of Fig. 8 that a line feature is detected in the 2001 observation, while it does not seem to be present in the 2002 observation (same figure). The statistics of the 1997 observation are too poor to give conclusive results.

On the other hand, after fitting the continuum of the 2001 data with the Compton reflection model, the residuals no longer show clear evidence of a line feature.
We thus concentrate on the 2001 best fit observation and add a Gaussian emission line. The fit is improved $\left(\Delta \chi^{2}=6.07\right.$ for 1 more parameter; F-test 5.39 and probability $\left.2.29 \times 10^{-2}\right)$ with a line at $6.53 \mathrm{keV}$ consistent with neutral $\mathrm{Fe} \mathrm{K} \alpha$ and weakly ionized species. Note that the line is not resolved as $\sigma_{\mathrm{Fe}}<500 \mathrm{eV}$ is smaller than the MECS energy resolution.

Both Compton reflection from cold material and the thermal Comptonisation model give a line energy of $6.53 \mathrm{keV}$ but the latter gives a slightly larger equivalent width. According to George \& Fabian (1991), if a reflection is present, it has to be associated with an Fe line. In the neutral case, they computed an equivalent width of the order of $1.7 \mathrm{keV}$ with respect to the reflection or $\sim 150 \mathrm{eV}$ with respect to the continuum, for $R=1$ (see also Zdziarski et al. 2002). Our measures of the line equivalent width and $R$ are inconsistent with the predictions of George \& Fabian (1991). We shall discuss in Sect. 4 a possible reason for this deviation.

Unlike Reeves et al. (2001) and Page et al. (2003), no residuals are found at $\sim 7 \mathrm{keV}$, but the line equivalent width and centroid suggest that it could also be a blend of a neutral narrow component and a weakly ionized one. Adding a second Gaussian to the model while freezing the line energies to $6.4 \mathrm{keV}$ and $6.7 \mathrm{keV}$ (rest frame) following Reeves et al. (2001) was not a significant change; the same applies to the relativistically-broadened accretion disk line model (Fabian et al. 1989) which was preferred by Reeves et al. (2001).

For the 2002 observation, the residuals were not improved when using the Compton reflection model and thus were identical to Fig. 8.

\subsection{Line feature at $1 \mathrm{keV}$}

The spectrum of the 2002 observation contains a narrow emission line feature centered at about $1 \mathrm{keV}$ (see Fig. 8, lower right panel). We have fitted a model composed of a power law plus Galactic absorption and a Gaussian component (Fig. 12) and found that the line is centered at $0.98_{-0.34}^{+0.08} \mathrm{keV}$ (quasar rest frame), has a width of $0.11_{-0.11}^{+0.33} \mathrm{keV}$ (not resolved; the LECS energy resolution at $1 \mathrm{keV}$ is $\sim 0.2 \mathrm{keV}$ ) and an equivalent width of $518 \mathrm{eV}$. The addition of the line significantly improves the fit at better than the $98 \%$ confidence level according to the F-test. Note however that even if this simple model allows us to estimate the line centroid energy and total flux, it is physically unrealistic as it is likely that this line results from 
Table 5. Fe K $\alpha$ line: fits of the 2001 observation, MECS data only. A stands for photoelectric absorption (fixed to the Galactic value), PEXRAV for the Compton reflection from cold material, COMPHA for the thermal Comptonisation model and $\mathrm{G}$ for Gaussian (line energies are rest frame). $R, E_{\mathrm{c}}, k T_{\mathrm{e}} / m_{\mathrm{e}} c^{2}$ and $\tau$ were fixed to their best fit values of Table 3 .

\begin{tabular}{lcccccc}
\hline \hline Model & $\Gamma$ & $E_{\mathrm{Fe}}$ & $\sigma_{\mathrm{Fe}}$ & $E W_{\mathrm{Fe}}$ & $\chi^{2}$ (d.o.f.) & Proba. \\
\hline A+PEXRAV & $1.99_{-0.02}^{+0.03}$ & $\mathrm{keV}$ & $\mathrm{eV}$ & $\mathrm{eV}$ & & \\
A+PEXRAV+G & $1.99^{f}$ & $6.53_{-0.19}^{+0.23}$ & $<447.60$ & 56.80 & $86.74(77)$ & 0.210 \\
A+COMPHA & - & - & - & - & $95.53(79)$ & 0.099 \\
A+COMPHA+G & - & $6.53_{-0.16}^{+0.16}$ & $<314.82$ & 71.90 & $86.25(77)$ & 0.220 \\
\hline
\end{tabular}

${ }^{f}$ Parameters fixed in the fit.

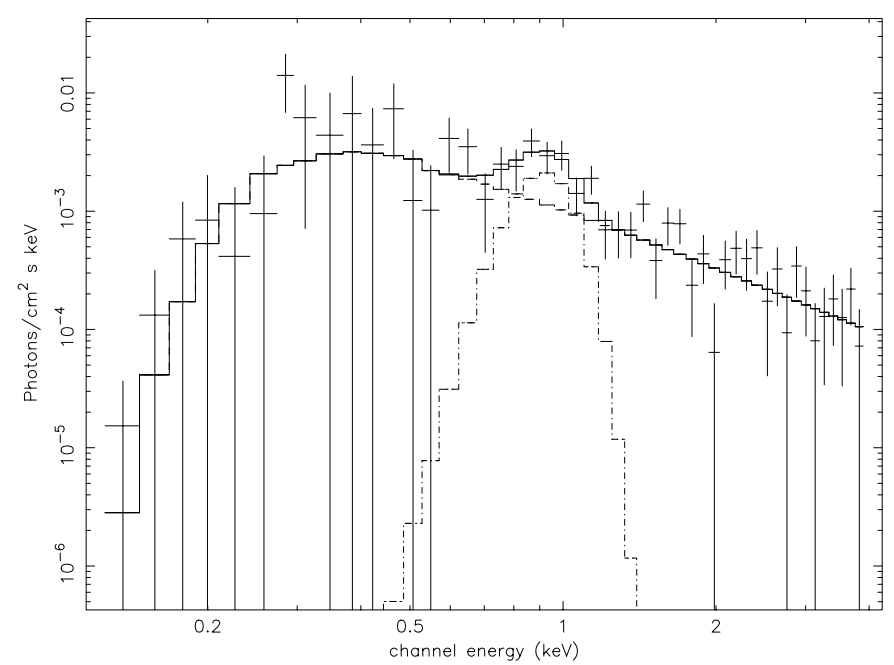

Fig. 12. Observation taken in 2002, LECS data only, unfolded spectrum fitted with a power law plus Galactic absorption and a Gaussian component centered at an energy of about $1 \mathrm{keV}$.

a blend of several emission lines, predominantly from ionized species of $\mathrm{Ne}$ and possibly from the Fe $\mathrm{L}$ shell.

A similar feature was also found in the ASCA spectrum of the quasar PG 1244+026 (Fiore et al. 1998; George et al. 2000) as well as in the ASCA spectrum of the Seyfert 1 galaxies TON S180 (Turner et al. 1998) and Akn 564 (Turner et al. 1999).

We searched the XMM-Newton data for this feature but did not find clear evidence of it (the equivalent width reached a maximum of $10 \mathrm{eV}$ ).

\section{Discussion and conclusion}

The nature of Mrk 205. It has been noted that, in contrast to the Seyfert 1 case, the Fe K $\alpha$ line in quasars was more seldom detected and that if detected, the line energy was around $6.7 \mathrm{keV}$ instead of $6.4 \mathrm{keV}$ (Reeves \& Turner 2000). It was thus suggested that sensitive instruments like the EPIC camera on $X M M$-Newton could observe the Fe $\mathrm{K} \alpha$ emission of the quasar in detail. From our X-ray observations of Mrk 205, and from the luminosity we obtained, we suggest caution in classifying this object. Its luminosity is found in the region in which it is difficult to distinguish between a Seyfert 1 or a quasar. It seems thus difficult to generalize any findings made on this object to a whole class.

The soft excess variability. The soft excess seems to be variable in this source although a very strong soft excess was never reported. It seems to be absent/not detected in 1997, strong in 2000, weak in 2001 and absent again in 2002 (thus varied over a 2 week time scale). The variability of the soft excess cannot be associated with variability of a cold absorber as we have shown in Fig. 1 and Sect. 3 that the absorbing column density deduced from spectral modeling was roughly constant over all the observations.

Note that the soft excess component measured here should not be associated with direct thermal emission from the accretion disk because its temperature is much too high.

Thermal Comptonisation. The historic variations of the photon index can be explained in the framework of thermal Comptonisation. Mrk 205 showed large 2-10 keV flux variations between the 1997 (high flux state), 2000 (low flux state), 2001 (high flux state) and 2002 (low flux state) observations which do not seem to be correlated with variability of the soft excess.

However, in the thermal Comptonisation processes, the index of the Comptonised component is inversely correlated with the ratio of the photon seed flux to the Comptonised power law flux. We can test this hypothesis if we assume that the soft excess strength is proportional to the strength of the soft photon field (Walter \& Fink 1993; Walter \& Courvoisier 1990). The index for 2000 was $1.73 \pm 0.02$ for a ratio of $23 \%$ while for 2001 the index was $2.00 \pm 0.03$ for a ratio of $3 \%$. This is thus in disagreement with our expectations.

On the other hand, using the COMPHA model to estimate the UV flux, we found that an increase in the photon index is associated with a larger UV flux, in agreement with simple expectations of a thermal Comptonisation model. Similar conclusions were found by Petrucci et al. (2000) for the Seyfert 1 galaxy NGC 5548. The contradiction between the soft $\mathrm{X}$-ray and UV variations is not very surprising. Indeed the soft excess component is certainly linked to the UV bump through complex radiative transfer (e.g. Comptonisation in the warm (few keV) upper layer of the accretion disk). Consequently, its expected spectral behavior is not straightforward and may be at odds with the zeroth order expectations.

Constraints on the reflecting material. The nature of the reprocessing medium can be the accretion disk or some 
distant obscuring material. If the $\mathrm{Fe} \mathrm{K} \alpha$ line is produced in the inner part of the accretion disk, its profile should be considerably broader, with very distinct asymmetries due to Doppler and gravitational redshifts. This is not possible to test with the BeppoSAX data. Page et al. (2003) report that they did not find evidence for this in the XMM-Newton data.

The strength of the Compton reflection $R$ is systematically higher here than in Seyfert galaxies $(R \simeq 0.8 \pm 0.2$, Nandra \& Pounds 1994), while on the contrary, the iron line equivalent width is smaller than the mean $E W$ value. However our measures of $R$ are consistent with $R<1$ at a $90 \%$ confidence, see Fig. 9.

The inconsistency between the best fit values of $R$ and the line $E W$ may then occur by chance and the reflecting material in Mrk 205 may simply cover a solid angle $<2 \pi$ as seen from the X-ray source. The line energy then also suggests that the material is neutral. A low $\mathrm{Fe}$ abundance will make the line weaker and the Compton reflection hump stronger (as observed) by reducing the opacity above the Fe K edge (George \& Fabian 1991).

Given these constraints a simple solution could be that the reprocessing is done in a distant material e.g. the dust torus.

However, we cannot rule out the presence of close mildly ionized material. Indeed Nayakshin \& Kallman (2001) have shown that in the case of relatively strong illumination by local X-ray flares, the upper layer of the reflecting material may become strongly ionized. In this case the reflection features are mainly produced by the neutral depth layer and the expected line energy is $6.4 \mathrm{keV}$ as we observed. The line $E W$ is however smaller than the one expected in the case of a neutral reflector since the line may be partly Comptonised in the hot skin. Clearly this skin cannot be highly ionized since it would act like a perfect mirror and the reflection hump would be suppressed, unlike what we observe. Lines in the soft band are also expected in the case of a mildly ionized medium and it may explain the poor fit of the soft excess by a simple black body component.

The 1 keV line. The spectrum of January 2002 contains a narrow emission feature centered at an energy of $\sim 1 \mathrm{keV}$ which was also found in at least 3 other AGN. This feature could be explained by a blend of several emission lines, predominantly from ionized species of $\mathrm{Ne}$ and possibly from the Fe L shell. The line emission could be a result of reflection in photoionized matter. It could also result from recombination in an optically thin thermal plasma.

Acknowledgements. P.F. acknowledges a grant from the Swiss National Science Foundation. This research has made use of the NASA/IPAC Extragalactic Database (NED) which is operated by the Jet Propulsion Laboratory, California Institute of Technology, under contract with the NASA. This research has also made use of the SIMBAD database, operated at CDS, Strasbourg, France.

\section{References}

Arp, H. 1996, A\&A, 316, 57

Bahcall, J. N., Jannuzzi, B. T., Schneider, D. P., et al. 1992, ApJ, 398, 495

Beckmann, V., Wolter, A., Celotti, A., et al. 2002, A\&A, 383, 410
Boella, G., Butler, R. C., Perola, G. C., et al. 1997a, A\&AS, 122, 299

Boella, G., Chiappetti, L., Conti, G., et al. 1997b, A\&AS, 122, 327

Bowen, D. V., Pettini, M., Penston, M. V., \& Blades, C. 1991, MNRAS, 248, 153

Brunner, H., Mueller, C., Friedrich, P., et al. 1997, A\&A, 326, 885

Ciliegi, P., \& Maccacaro, T. 1996, MNRAS, 282, 477

Condon, J. J., Cotton, W. D., Greisen, E. W., et al. 1998, AJ, 115, 1693

Della Ceca, R., Palumbo, G. G. C., Persic, M., et al. 1990, ApJS, 72, 471

Dickey, J. M., \& Lockman, F. J. 1990, ARA\&A, 28, 215

Fabian, A. C., Rees, M. J., Stella, L., \& White, N. E. 1989, MNRAS, 238,729

Fiore, F. 1994, in The Soft X-ray Cosmos, ed. Schlegel, \& Petre, AIP Conf. Proc., 313, 95

Fiore, F., Elvis, M., McDowell, J. C., et al. 1994, ApJ, 431, 515

Fiore, F., Matt, G., \& Nicastro, F. 1997, MNRAS, 284, 731

Fiore, F., Matt, G., Cappi, M., et al. 1998, MNRAS, 298, 103

Fiore, F., Guainazzi, M., \& Grandi, P. 1999, http://www . asdc.asi.it/bepposax/software/index.html

Frontera, F., Costa, E., \& Dal Fiume, D. 1997, A\&AS, 122, 357

George, I. M., \& Fabian, A. C. 1991, MNRAS, 249, 352

George, I. M., Turner, T. J., Yaqoob, T., et al. 2000, ApJ, 531, 52

Guainazzi, M., \& Matteuzzi, A. 1997, Technical report, ftp://www.asdc.asi.it/pub/sax/doc/reports/ sdc-tr014.ps.gz

Haardt, F. 1993, ApJ, 413, 680

Haardt, F., Maraschi, L., \& Ghisellini, G. 1997, ApJ, 476, 620

Huchra, J. P., Geller, M. J., Henry, J. P., \& Postman, M. 1990, ApJ, 365,66

Lightman, A. P., \& White, T. R. 1988, ApJ, 335, 57

Magdziarz, P., \& Zdziarski, A. A. 1995, MNRAS, 273, 837

McDowell, J. C., Elvis, M., Wilkes, B. J., et al. 1989, ApJ, 345, L13

Morrison, R., \& McCammon, D. 1983, ApJ, 270, 119

Nandra, K., \& Pounds, K. A. 1994, MNRAS, 268, 405

Nandra, K., George, I. M., Mushotzky, R. F., et al. 1997, ApJ, 488, L91

Nayakshin, S., Kazanas, D., \& Kallman, T. R. 2000, ApJ, 537, 833

Nayakshin, S., \& Kallman, T. R. 2001, ApJ, 546, 406

Page, M. J., Davis, S. W., \& Salvi, N. J. 2003, MNRAS, 343, 1241

Parmar, A. N., Martin, D. D. E., Bavdaz, M., et al. 1997, A\&AS, 122, 309

Petrucci, P. O., Haardt, F., Maraschi, L., et al. 2000, ApJ, 540, 131

Reeves, J. N., \& Turner, M. J. L. 2000, MNRAS, 316, 234

Reeves, J. N., Turner, M. J. L., Pounds, K. A., et al. 2001, A\&A, 365, L134

Rush, B., Malkan, M. A., \& Edelson, R. A. 1996a, ApJ, 473, 130

Rush, B., Malkan, M. A., Fink, H. H., \& Voges, W. 1996b, ApJ, 471, 190

Singh, K. P., Rao, A. R., \& Vahia, M. N. 1991, A\&A, 248, 37

Stockton, A., Wyckoff, S., \& Wehinger, P. A. 1979, ApJ, 231, 673

Tananbaum, H., Avni, Y., Branduardi, G., et al. 1979, ApJ, 234, L9

Turner, T. J., George, I. M., \& Nandra, K. 1998, ApJ, 508, 648

Turner, T. J., George, I. M., Netzer, H., et al. 1999, ApJ, 526, 52

Véron-Cetty, M. P., \& Véron, P. 2001, A\&A, 374, 92

Walter, R., \& Courvoisier, T. J.-L. 1990, A\&A, 233, 40

Walter, R., \& Fink, H. H. 1993, A\&A, 274, 105

Weedman, D. W. 1970, ApJ, 161, L113

White, T. R., Lightman, A. P., \& Zdziarski, A. A. 1988, ApJ, 331, 939

Williams, O. R., Turner, M. J. L., Stewart, G. C., et al. 1992, ApJ, 389, 157

Wilkes, B. J., \& Elvis, M. 1987, ApJ, 323, 243

Womble, D. S. 1993, PASP, 105, 1043

Zamorani, G., Henry, J. P., Maccacaro, T., et al. 1981, ApJ, 245, 357

Zdziarski, A. A., Leighly, K. M., Matsuoka, M., et al. 2002, ApJ, 573, 505 